\title{
Comparative transcriptomic analyses of four Phalaenopsis species to identify and characterize the WUSCHEL-related homeobox (WOX) gene family
}

\author{
MADHVI KANCHAN, JASPREET K. SEMBI * \\ Department of Botany, Panjab University, Chandigarh, India
}

\begin{abstract}
In the present study, we identified and characterized the plant-specific WUSCHEL -related homeobox (WOX) gene family that plays a major role in the determination of cell fate, early embryonic pattern formation, stem cell maintenance, organogenesis, flower development and somatic embryogenesis. For this purpose, the transcriptomes of four species of Phalaenopsis, namely $P$. aphrodite, $P$. modesta, $P$. bellina and $P$. lueddemanniana, were analysed, and nine $P a W O X$, ten $P m W O X$, eight $P b W O X$ and nine $P I W O X$ transcripts were identified. The duplication event analysis showed the presence of two duplication events in $P$. lueddemanniana, one each in $P$. bellina and $P$. modesta and no duplication event in $P$. aphrodite. During the evolutionary analysis, all the WOX proteins were clustered with those of Arabidopsis thaliana (AtWOX), Phalaenopsis equestris(PeWOX), Apostasia shenzhenica and Dendrobium catenatum (DcWOX). The expression analysis of the WOX genes suggested their critical role in floral development and in other developmental processes. The secondary and tertiary structural analysis of seven selected WOX proteins was then performed, with each protein representing its respective clade. The results provide a valuable resource for further studies of the molecular mechanisms of floral and vegetative developments in Phalaenopsis species.
\end{abstract}

Key words: WUSCHEL, WOX, expression analysis, Phalaenopsis, orchids

\section{Introduction}

The WUSCHEL-related homeobox (WOX) gene family is a subgroup of the homeobox transcription factor superfamily containing the conserved domain of 60-66 amino acid helix-loop-helix-turn-helix structure (Gehring et al., 1994). It controls cell fate determination, early embryonic pattern formation, stem cell maintenance, organogenesis, flower development, somatic embryogenesis and stress tolerance in plants (Deveaux et al., 2008; Costanzo et al., 2014; Jha et al., 2020). The WOX gene family was first identified in Arabidopsis thaliana, where the WUSCHEL (WUS) gene of modern clade was isolated and functionally characterized (Laux et al., 1996; Haecker et al., 2004). In Arabidopsis, the WOX gene family consists of 15 members and is divided into three distinct clades: WUS (WUS and WOX 1-7), intermediate (WOX8, WOX9, WOX11 and WOX12) and an- cient (WOX10, WOX13 and WOX14) (van der Graaff et al. 2009). The WUS clade is a modern clade restricted to angiosperms, the intermediate clade belongs to the intermediate plant groups such as pteridophytes and gymnosperms, and the ancient clade is present among all plant groups from green algae to angiosperms (van der Graaff et al., 2009; Lian et al., 2014). The WUS gene plays a role in the maintenance of shoot apical meristem in Arabidopsis (Zuo et al., 2002), somatic embryogenesis in Gossypium hirsutum (Bouchabké-Coussa et al., 2013) and organogenesis in Coffea canephora (ArroyoHerrera et al., 2008). WUS also regulates floral development by acting as an activator of the AGAMOUS gene in flowers (Lohmann et al., 2001). The other members of the WUS clade contribute to different stages of plant development; AtWOX5 performs the same function as AtWUS in root and shoot apical meristems (Oshchep-

\footnotetext{
* Corresponding author: Department of Botany, Panjab University, Chandigarh, India; e-mail: jaspreet.sembi@pu.ac.in
} 
kova et al., 2017). The WOX3 (PRESSED FLOWER or $P R S$ ) gene of $A$. thaliana regulates the development of lateral sepals, stamens and stipules (Matsumoto and Okada, 2001), whereas its ortholog in Zea mays, NARROW SHEATH(NS), enhances the growth of the leaf sheath and the proximal blade region (Scanlon et al., 1996; Nardmann et al., 2004). The WOX4 gene regulates vascular cell differentiation through the auxin-dependent pathway for the lateral growth of plants (Suer et al., 2011). AtWOX6 (PRETTY FEW SEEDS2 or PFS2) is required for ovule development and pattern formation (Park et al., 2005). AtWOX7 plays the central integrating role in lateral root development and inhibits lateral root development in response to plant sugar status (Kong et al., 2016). WOX2 was also found to be involved in somatic embryogenesis in Larix decidua (Rupps et al., 2016). The WOX2, WOX8 and WOX9 genes of Arabido$p$ sis play a critical role in early embryo development where they act as an essential cell fate regulator and show the highest functional redundancy (Breuninger et al., 2008; Ueda et al., 2011). $W O X 8$ and $W O X 9$ are known as STIMPY (WOX9) and STIMPY-LIKE (WOX8) genes in A. thaliana and EVERGREEN (EVG) and SISTER OF EVERGREEN (SOE) in Petunia; they are essential for inflorescence development (Rebocho et al., 2008; Costanzo et al., 2014). WOX11 and its homolog WOX12 play an essential role in de novo root organogenesis (Liu et al., 2014). WOX13 of ancient clade shows zestful expression during various developmental processes such as primary lateral root initiation, gynoecium and embryo development, floral transitions and vegetative fruit development (Deveaux et al., 2008; Romera-Branchat et al., 2013).

The current study was performed to characterize the WOX genes in Phalaenopsis orchids. Orchids are economically important plants renowned for their spectacular flowers with high longevity. Phalaenopsis is a tropical epiphytic orchid accounting for nearly $80 \%$ of the orchid trade in floriculture industry (Wu et al., 2012). This genus comprises nearly 70 species, of which $P$. aphrodite, $P$. modesta, $P$. bellina and $P$. lueddemanniana were selected for the present study. This study was conducted to identify and characterize the WOX genes in different Phalaenopsis species with a particular focus on the evolutionary analysis and expression profiling.

\section{Materials and methods}

\section{Identification of the WOX family protein}

To identify the WOX proteins of $P$. aphrodite $(\mathrm{Su}$ et al., 2011), $P$. bellina, $P$. modesta, and $P$. lueddemanniana, the TBLASTN search was performed in Orchidstra 2.0 database (http://orchidstra2.abrc.sinica.edu.tw/ orchidstra2/index.php; Chao et al., 2017). The WOX protein sequences of $A$. thaliana (AtWOX) (van der Graaff et al., 2009), Phalaenopsis equestris (PeWOX) and Dendrobium catenatum (DcWOX) (Ramkumar et al., 2018) were used as queries against four species of Phalaenopsis. The presence of a homeobox domain (pfam00046) was verified using SMART (http://smart.embl-heidelberg.de/; Schultz et al., 2000) tools.

\section{Conserved domain and motif analysis}

The conserved domain (homeobox-domain) was identified using Expasy - Prosite (https://prosite. expasy.org/; Sigrist et al., 2012). MULTALIN (http://multalin.toulouse.inra.fr/multalin/; Corpet 1988) was used to identify the location of the DNA binding helix-loop-helix-turnhelix region. The MEME suite online server (http:// meme-suite.org/tools/meme; Bailey et al., 2009) was used to identify the conserved motifs, with pre-set parameters (maximum number of motifs: 05 , number of repetitions: any, optimal motif width: $\geq 6$ and $\leq 150$ ).

\section{Physicochemical characterization}

The molecular weight, aliphatic index, instability index, pI and grand average of hydropathicity (GRAVY) were calculated using the Expasy Protparam tool (https://web.expasy.org/protparam/; Gasteiger et al., 2005). The online tools CELLO v.2.5 (http://cello.life. nctu.edu.tw/; Yu et al. 2006) and WoLF PSORT (https:// www.genscript.com/wolf-psort.html; Horton et al., 2007) were used to predict the subcellular location of the WOX proteins. The presence of a signal peptide and the transmembrane helix was predicted using the online tools SignalP.4.0 (http://www.cbs.dtu.dk/services/signalp/; Petersen et al., 2011) and TMHMM v.2.0 (http:// www.cbs.dtu.dk/services/TMHMM/; Krogh et al., 2001).

\section{Duplication events and ortholog prediction}

The NCBI BLASTp search was performed to predict ortholog sequences of all four species of Phalaenopsis against closely related orchids, $P$. equestris (PeWOX), 


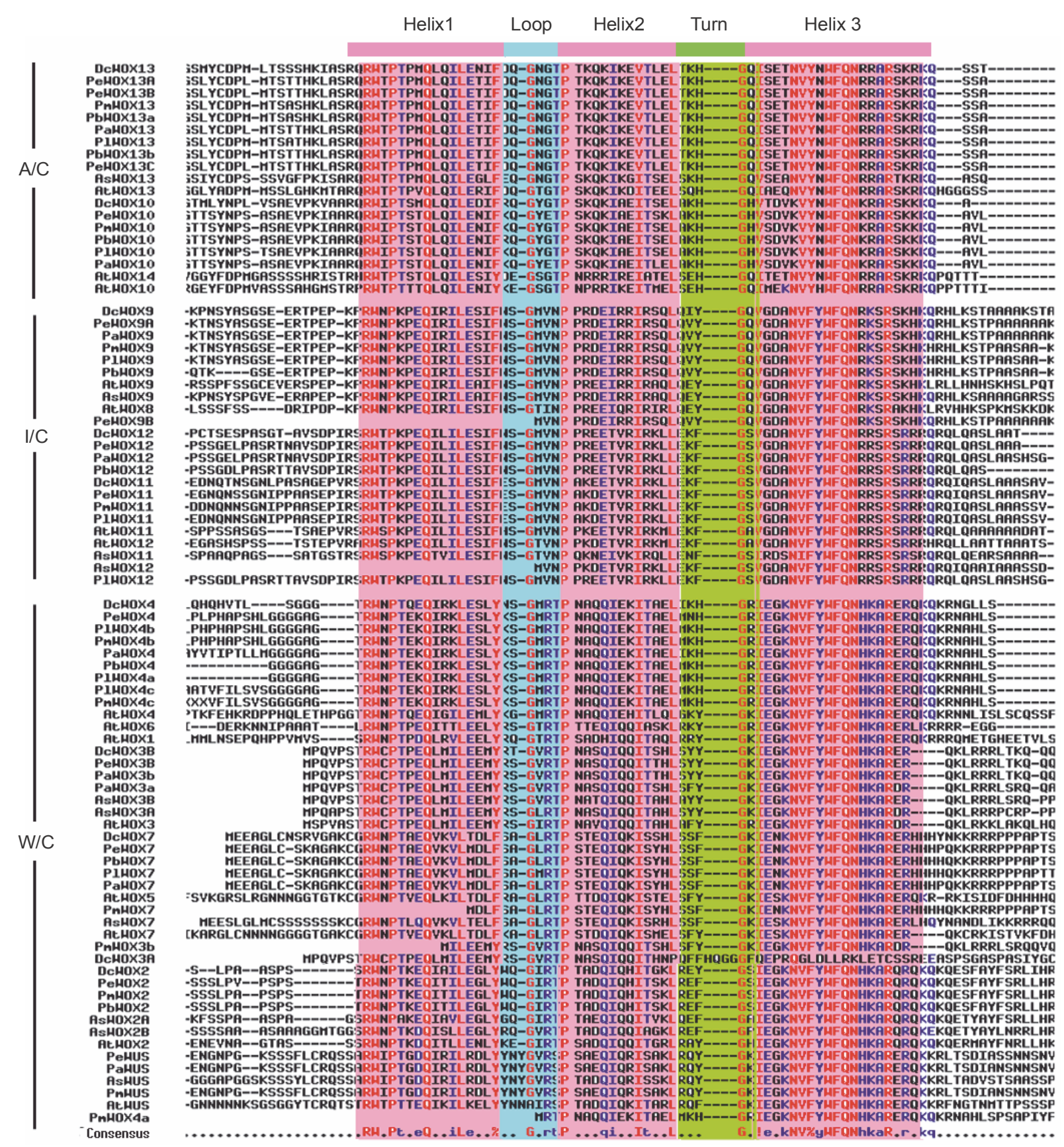

Fig. 1. Multiple sequence alignment of WOX protein sequences from $P$. bellina, $P$. modesta, $P$. aphrodite and $P$. lueddemanniana with $P$. equestris, $D$. catenatum, $A$. shenzhenica and $A$. thaliana shows homeodomain with the helix-loop-helix-turn-helix region, which is a characteristic of the WOX protein family (highly conserved amino acid sequence regions are shown in red and the less conserved ones are indicated in blue); the helix regions are marked in pink, the loop region in sky blue and the turn region in green

D. catenatum (DcWOX), A. shenzhenica (AsWOX) and the model plant $A$. thaliana (AtWOX), where each WOX protein query was independently blasted against WOX protein sequences of the target species. The percentage identity matrix of $W O X$ gene sequences was analysed on the basis of the alignment of CDS sequences using MUSCLE (https://www.ebi.ac.uk/Tools/msa/muscle/;
Edgar, 2004) server. More than 80\% identity was shared by duplicated genes at the nucleotide level.

\section{Phylogenetic analysis}

The full-length and conserved homeodomain sequences of the putative PaWOX, PmWOX, PIWOX and $\mathrm{PbWOX}$ family members were aligned with the MUSCLE 

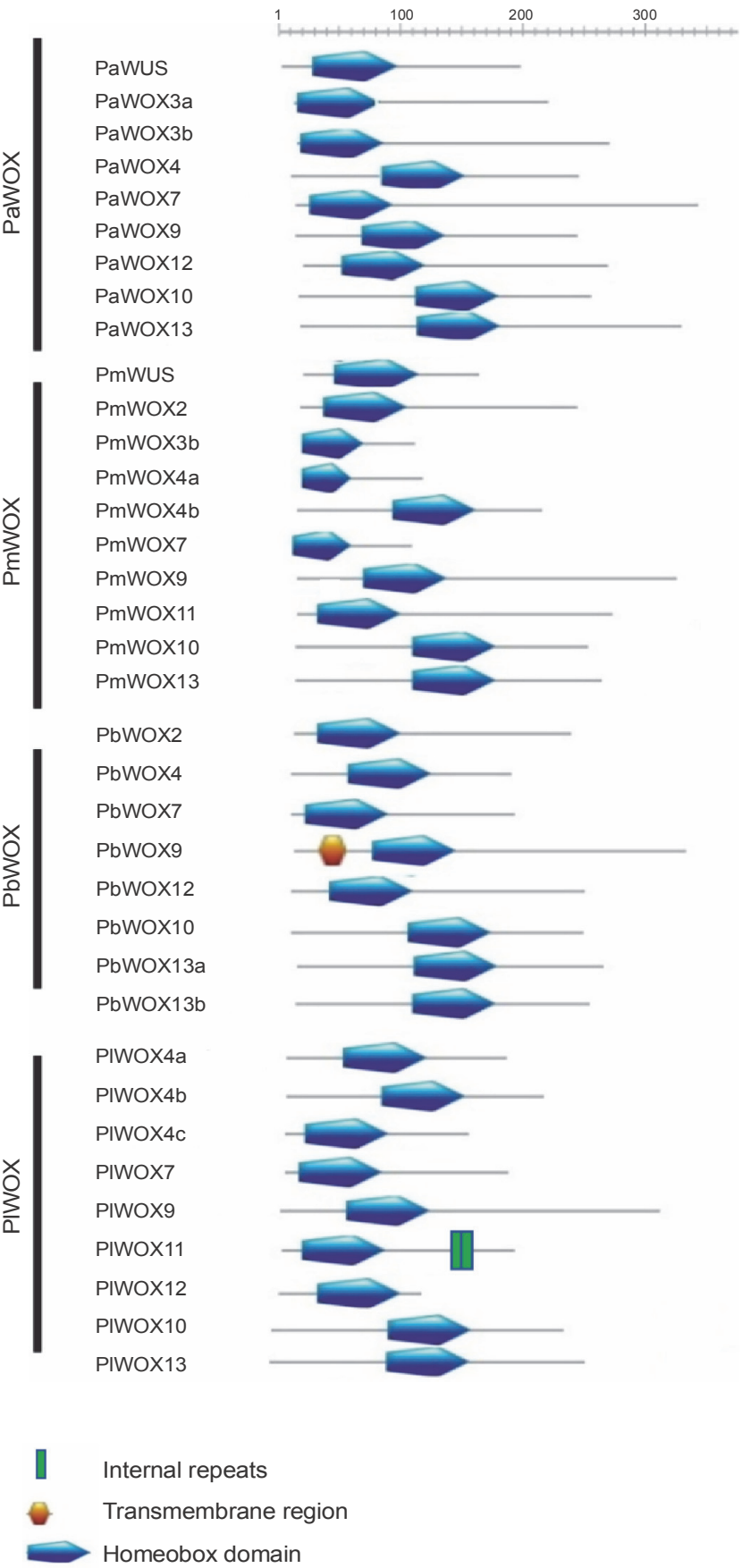

Fig. 2. The domain architecture analysis shows a homeobox domain for PaWOX, PmWOX, PbWOX and PIWOX protein sequences

program. A phylogenetic tree of the WOX protein sequences from six plant species (A. thaliana, $P$. equestris, $P$. bellina, $P$. modesta, $P$. aphrodite, $P$. lueddemanniana, $A$. shenzhenica and $D$. catenatum) was constructed based on the maximum likelihood method using the MEGA7 tool (http://www.megasoftware.net/; Kumar et al., 2016) with 1000 iterations for the bootstrap values.

\section{Expression analysis}

The CDS sequences of the WOX genes of all Phalaenopsis species were used as a query during the BLASTn search against the high-throughput RNA-seq data generated (NCBI-SRA database) from different developmental stages such as roots (SRX2439763), leaf (SRX2439762), fully opened flower (SRX2439761), large flower bud (SRX2196321) and small flower bud (SRX2439759) of P. aphrodite; small bud (SRX2210819), large bud (SRX2210818) and fully opened flower (SRX2210817) of P. modesta; root (SRX2210822), leaf (SRX2210823), large flower bud (SRX2210821) and fully opened flower (SRX2210820) of $P$. bellina; and root (SRX2210813), leaf (SRX2210814), large bud (SRX2210811), small bud (SRX2210812) and fully opened flower (SRX2210810) of P. lueddemanniana (https://blast.ncbi.nlm.nih.gov/Blast. cgi?PROGRAM=blastn\&PAGE_TYPE=BlastSearch\&B LAST_SPEC=SRA\&LINK_LOC=blasttab), and the hit counts were noted. The RPKM (reads per kilobase per million) values were calculated using the formula [RPKM $=(C \times 109) /(N \times L)$, where $N$ is the total mapped reads in the RNA-seq experiment concerned, $L$ is the base-pair length of the gene, and $C$ is the number of hits for the candidate gene)] (Mortazavi et al., 2008). The heat maps were generated using the Hierarchical Clustering Explorer 3.5 (http://www.cs.umd.edu/hcil/hce/; Seo et al. 2006).

\section{Secondary structures of WOX proteins}

Secondary structures (alpha-helices, random coils, beta turns and extended strands) for the selected WOX protein sequences were predicted using the SOPMA (self-optimized prediction method with alignment), a secondary structure prediction tool (https://npsa-prabi. ibcp.fr/cgi-bin/npsa_automat.pl?page=/NPSA/npsa_ sopma.html; Sapay et al., 2006). The tool includes the homologue method that takes information from an alignment of sequences belonging to the same protein family.

\section{$3 D$ structure prediction and analysis of WOX proteins}

The molecular modelling tool I-TASSER (https:// zhanglab.ccmb.med.umich.edu/I-TASSER/; Yang et al., 2015) was used to predict the tertiary structure using top 10 homologues available in the protein data bank (PDB) with specific parameters [c-scores (confidence score for estimating the quality of the models predicted by I-TASSER), BS-score, TM-scores and IDEN coverage 


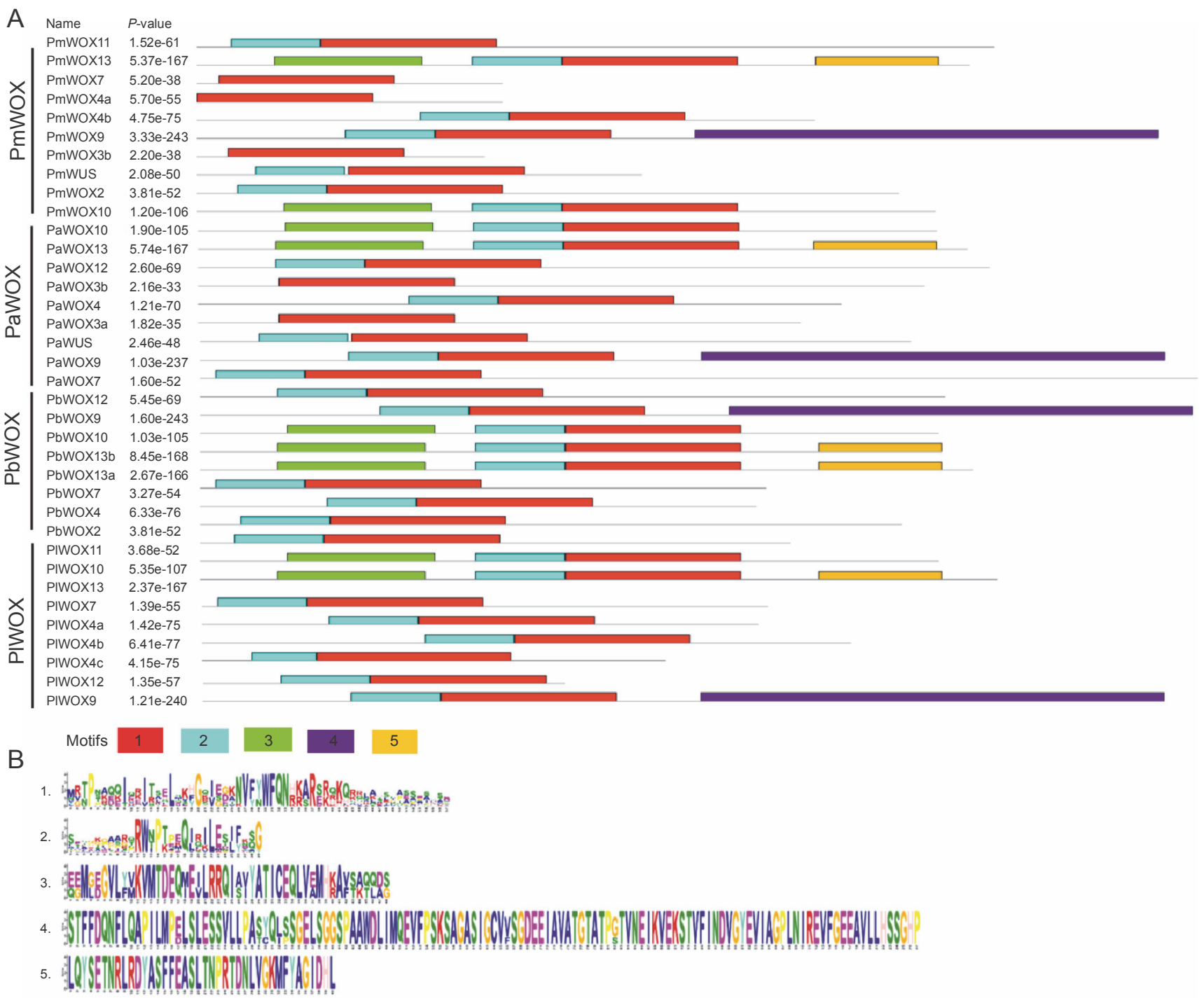

Fig. 3. The motif analysis for PaWOX, PmWOX, PbWOX and PIWOX: A) conserved motifs in sequences are marked in coloured boxes, B) sequence logo of WOX protein motifs

of the alignment by TM-align]. The binding site prediction with the BS-score value of $>0.5$ was considered as highly confident. The molecular viewer PyMOL (https://pymol.org/; DeLano 2002) was used to visualize the $3 \mathrm{D}$ protein structure.

\section{Results}

\section{Protein identification and domain analysis}

In the present study, we identified and characterized 36 WOX genes from $P$. bellina, $P$. modesta, $P$. aphrodite and $P$. lueddemanniana. The nomenclature of proteins and their respective genes was performed following their closest phylogenetic homologs in $A$. thaliana,
$P$. equestris and D. catenatum (van der Graa et al., 2009; Ramkumar et al., 2018). All the protein sequences identified contained a WUSCHEL-related homeobox domain. The multiple sequence alignment showed the occurrence of the conserved helix-loop-helix-turn-helix region in homeodomain-encoded WOX proteins (Fig. 1). One transmembrane region was found in $\mathrm{PbWOX} 9$, and two internal repeats were found in PIWOX11 (Fig. 2). The maximum number of motifs was found in the ancient clade, whereas the least number of motifs was identified in the WUS clade (Fig. 3). Motifs 1 and 2 were present in all WOX proteins and were predicted to encode the homeodomain. Members of the modern and intermediate clades had similar motif compositions, 
Table 1. Physiochemical characterization of WOX protein family

\begin{tabular}{|c|c|c|c|c|c|c|c|c|c|}
\hline $\begin{array}{l}\text { Protein } \\
\text { name }\end{array}$ & $\begin{array}{c}\text { Length } \\
\text { [aa] }\end{array}$ & $\begin{array}{c}\text { MW } \\
{[\mathrm{kDa}]}\end{array}$ & IP & INS & $\mathrm{AI}$ & GRAVY & Localization & $\mathrm{Sp}$ & TMD \\
\hline \multicolumn{10}{|c|}{ Phalaenopsis aphrodite } \\
\hline PaWUS & 230 & 25832.44 & 5.99 & 57.57 & 56.04 & -0.834 & nuclear & no & 0 \\
\hline PaWOX3a & 195 & 21906.56 & 8.63 & 73.86 & 58.05 & -0.756 & nuclear & no & 0 \\
\hline PaWOX3b & 236 & 26956.41 & 6.52 & 81.90 & 61.14 & -0.639 & nuclear & no & 0 \\
\hline PaWOX4 & 208 & 23454.73 & 10.01 & 67.30 & 72.26 & -0.766 & nuclear & no & 0 \\
\hline PaWOX7 & 329 & 37804.14 & 6.44 & 48.52 & 71.43 & -0.592 & cytoplasm & no & 0 \\
\hline PaWOX9 & 312 & 33887.09 & 6.8 & 51.26 & 77.24 & -0.325 & nuclear & no & 0 \\
\hline PaWOX12 & 256 & 28069.26 & 5.61 & 69.40 & 72.38 & -0.304 & nuclear & no & 0 \\
\hline PaWOX10 & 239 & 27208.62 & 5.72 & 53.71 & 66.19 & -0.644 & nuclear & no & 0 \\
\hline PaWOX13 & 249 & 28559.01 & 5.23 & 59.81 & 64.26 & -0.781 & nuclear & no & 0 \\
\hline \multicolumn{10}{|c|}{ Phalaenopsis modesta } \\
\hline PmWUS & 144 & 16681.78 & 9.63 & 57.43 & 62.36 & -0.750 & nuclear & no & 0 \\
\hline PmWOX2 & 227 & 25638.89 & 6.71 & 89.12 & 63.66 & -0.705 & nuclear & no & 0 \\
\hline PmWOX3b & 93 & 11020.54 & 10.21 & 78.64 & 60.75 & -0.823 & nuclear & no & 0 \\
\hline PmWOX4a & 99 & 11713.19 & 6.84 & 53.56 & 63.13 & -1.186 & nuclear & no & 0 \\
\hline PmWOX4b & 200 & 22624.71 & 9.75 & 62.84 & 66.40 & -0.800 & nuclear & no & 0 \\
\hline PmWOX7 & 99 & 11677.29 & 10.11 & 77.61 & 59.09 & -0.979 & nuclear & no & 0 \\
\hline PmWOX9 & 311 & 33931.17 & 6.83 & 51.15 & 77.78 & -0.358 & nuclear & no & 0 \\
\hline PmWOX11 & 258 & 27220.26 & 5.64 & 77.19 & 68.49 & -0.234 & nuclear & no & 0 \\
\hline PmWOX10 & 239 & 26979.48 & 5.85 & 54.68 & 69.41 & -0.544 & nuclear & no & 0 \\
\hline PmWOX13 & 250 & 28646.06 & 5.16 & 64.12 & 61.68 & -0.814 & nuclear & no & 0 \\
\hline \multicolumn{10}{|c|}{ Phalaenopsis bellina } \\
\hline PbWOX2 & 227 & 25621.9 & 6.71 & 86.85 & 63.66 & -0.696 & nuclear & no & 0 \\
\hline PbWOX4 & 180 & 20529.26 & 9.76 & 60.51 & 68.33 & -0.917 & nuclear & no & 0 \\
\hline PbWOX7 & 183 & 20937.99 & 9.18 & 59.00 & 71.42 & -0.650 & nuclear & no & 0 \\
\hline PbWOX9 & 321 & 35235.05 & 7.1 & 48.60 & 88.38 & -0.188 & plasma membrane & no & 1 \\
\hline PbWOX12 & 241 & 26531.48 & 5.44 & 73.83 & 69.59 & -0.412 & nuclear & no & 0 \\
\hline PbWOX10 & 239 & 27120.55 & 5.86 & 56.38 & 69.00 & -0.597 & nuclear & no & 0 \\
\hline PbWOX13a & 240 & 27438.72 & 5.44 & 64.21 & 62.21 & -0.807 & nuclear & no & 0 \\
\hline PbWOX13b & 250 & 28706.16 & 5.16 & 64.61 & 61.68 & -0.799 & nuclear & no & 0 \\
\hline \multicolumn{10}{|c|}{ Phalaenopsis lueddemanniana } \\
\hline PIWOX4a & 180 & 20486.19 & 9.76 & 63.91 & 66.17 & -0.932 & nuclear & no & 0 \\
\hline PIWOX4b & 210 & 23751.89 & 9.82 & 66.51 & 66.00 & -0.843 & nuclear & no & 0 \\
\hline PlWOX4c & 150 & 17134.28 & 9.45 & 60.99 & 65.07 & -0.988 & nuclear & no & 0 \\
\hline PIWOX7 & 183 & 21031.13 & 9.30 & 56.07 & 67.16 & -0.683 & nuclear & no & 0 \\
\hline PIWOX9 & 311 & 33935.16 & 6.83 & 51.43 & 78.10 & -0.348 & nuclear & no & 0 \\
\hline PIWOX11 & 191 & 19883.87 & 6.59 & 81.24 & 60.84 & -0.355 & nuclear & no & 0 \\
\hline PIWOX12 & 117 & 13123.66 & 11.05 & 73.83 & 66.75 & -0.779 & nuclear & no & 0 \\
\hline PIWOX10 & 239 & 27191.68 & 6.02 & 56.81 & 68.58 & -0.621 & nuclear & no & 0 \\
\hline PIWOX13 & 258 & 29538.14 & 5.34 & 65.51 & 63.18 & -0.776 & nuclear & no & 0 \\
\hline
\end{tabular}

* IP - isoelectric point, MW - protein molecular weight in kDa, INS - instability index, AI - aliphatic index, GRAVY - grand average of hydropathy, SP - signal peptide, TMD - transmembrane domain 
Table 2. Clade-wise distribution of WOX proteins of $P$. aphrodite, $P$. modesta, $P$. bellina and $P$. lueddemanniana

\begin{tabular}{l|c|c|c|c}
\hline \multicolumn{1}{c|}{ CLADES } & P. bellina & P. modesta & P. lueddemanniana & P. aphrodite \\
\hline \multirow{4}{*}{ Ancient } & PbWOX10 & PmWOX10, & PlWOX10 & PaWOX10 \\
& PbWOX13a & PmWOX13 & PIWOX13 & PaWOX13 \\
& PbWOX13b & & & \\
\hline \multirow{3}{*}{ Intermediate } & PbWOX12 & PmWOX11 & PIWOX11 & PaWOX12 \\
& PbWOX9 & PmWOX9 & PIWOX12 & PaWOX9 \\
& & & PlWOX9 & \\
\hline \multirow{3}{*}{ WUS } & PbWOX7 & PmWOX4a & PlWOX4a & PaWOX4 \\
& PbWOX4 & PmWOX4b & PIWOX4b & PaWOX3a \\
& PbWOX2 & PmWOX3b & PlWOX4c & PaWOX3b \\
& & PmWUS & PIWOX7 & PaWUS \\
& & PmWOX2 & & PaWOX7 \\
& & PmWOX7 & & \\
\hline
\end{tabular}
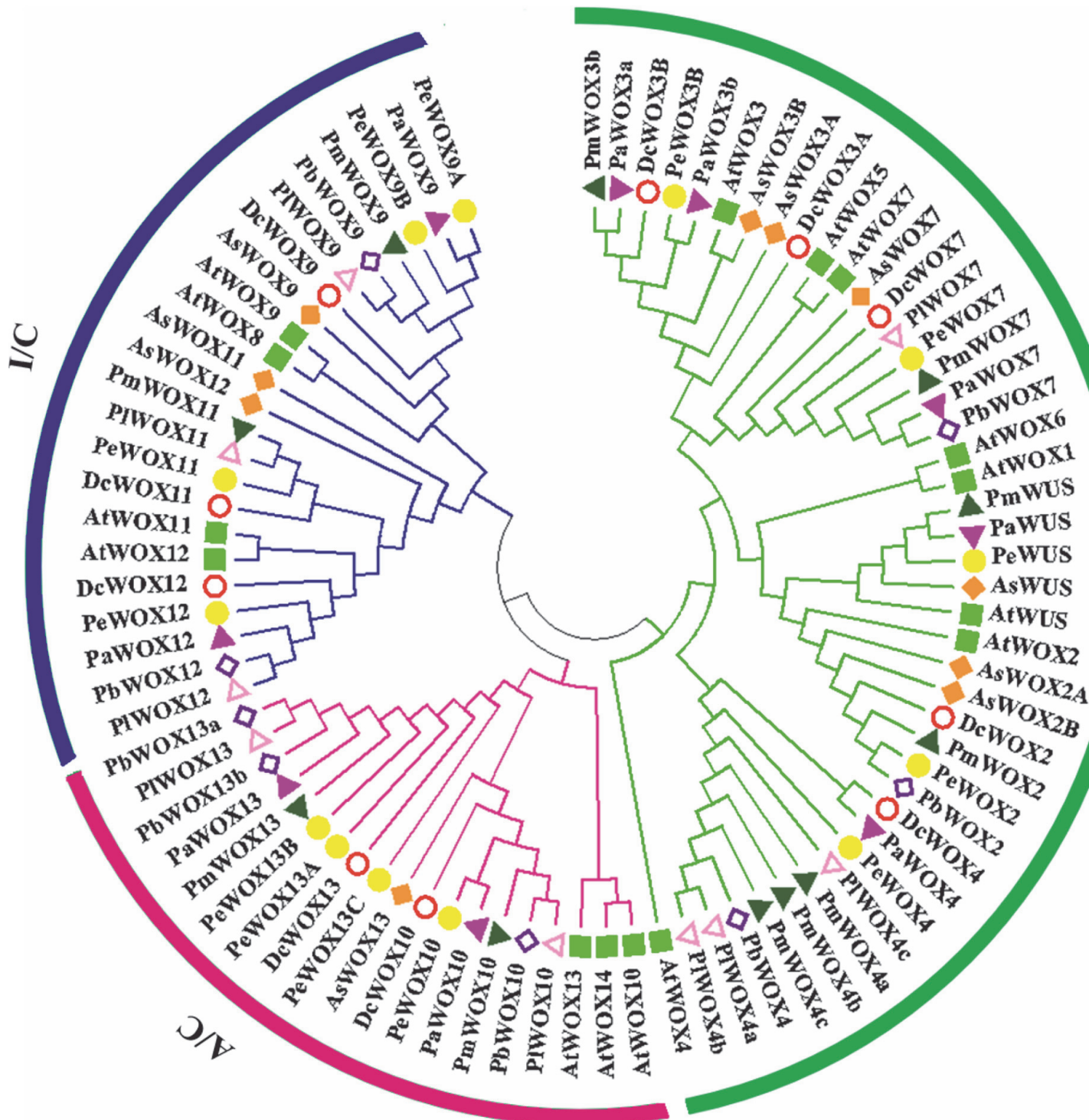

Fig. 4. The phylogenetic analysis of PaWOX, PmWOX, PbWOX and PIWOX proteins with AtWOX, PeWOX, AsWOX and DcWOX sequences shows clustering into ancient $(\mathrm{A} / \mathrm{C})$, intermediate $(\mathrm{I} / \mathrm{C})$ and WUS $(\mathrm{W} / \mathrm{C})$ clades (marked in pink, blue and green, 
except for motif 4 which was unique to the intermediate clade. On the other hand, motifs 3 and 5 were unique to the ancient clade (Fig. 3).

\section{Physicochemical analysis and topology study}

The average size of PaWOX, PbWOX, PIWOX and PmWOX was 250 aa (amino acid), 236 aa, 204 aa and 232 aa, respectively. The average molecular mass of PaWOX, PbWOX, PIWOX and PmWOX was $27.6 \mathrm{kDa}$, $26.4 \mathrm{kDa}, 23.24 \mathrm{kDa}$ and $20.6 \mathrm{kDa}$, respectively. The isoelectric point for all the WOX proteins ranged from 5.16 to 11.01 . The average values of the aliphatic index were 70.75, 69.29, 64.79 and 65.34 for PaWOX, PbWOX, PIWOX and PmWOX, respectively. A negative GRAVY value in all the sequences indicates that all WOX proteins were hydrophilic. All the proteins were found to be located in the nucleus, except for PbWOX9, which was predicted to be located in the plasma membrane, and PaWOX7, which was found in cytoplasm (Table 1).

\section{Evolutionary analysis}

The protein sequences of PaWOX, PmWOX, PbWOX and PIWOX were analysed along with 15 AtWOX, 14 PeWOX, 10 AsWOX (A. shenzhenica WOX) and 10 DcWOX sequences to predict their evolutionary relationship. A total of 36 protein sequences were clustered into three clades, namely ancient, intermediate and WUS, along with the respective members of AtWOX, PeWOX, AsWOX and DcWOX (Table 2, Fig. 4).

\section{Orthologs and duplication event analysis}

Orthologs counterparts for PaWOX, PmWOX, PbWOX and PIWOX protein sequences against AtWOX, PeWOX, DcWOX and AsWOX protein sequences were identified (Table $\mathrm{S} 1$ in the supplementary materials). Duplication events were predicted using $P a W O X, P m W O X, P b W O X$ and PIWOX CDS sequences. Two duplication events were predicted in $P$. lueddemanniana, and only one duplication event was predicted in $P$. bellina and $P$. modesta each, where $P b W O X 13 a$ and $P b W O X 13 b$ shared 98.06\% identity and PmWOX4b and PmWOX4a shared $86.19 \%$ identity, respectively. In $P$. lueddemanniana, PIWOX4C shared $96.64 \%$ identity with PIWOX4a and 96.84\% identity with PIWOX4b, and PIWOX4a and $P I W O X 4 b$ shared $99.63 \%$ identity. No duplication events were found in $P$. aphrodite (Table S2 in the supplementary materials).

\section{Expression profiling of the WOX genes}

The expression profiling of the $W O X$ genes was performed in tissues at various developmental stages (leaf, root, flower, small bud and large buds) in all the species of Phalaenopsis investigated ( $P$. bellina, $P$. modesta, $P$. aphrodite and $P$. lueddemanniana). The expression profile was determined by calculating the RPKM values (Table S3 in the supplementary materials) from hit counts in the NCBI-SRA database, and heat maps were then generated (Fig. 5). The expression of the WOX genes was found to be highly tissue-specific in all the four orchids. Most of the WOX genes were expressed in roots; however, this high expression was not replicated in other vegetative tissues, i.e. leaves. The expression of PbWOX2, PbWOX4, PIWOX4a, PIWOX4b, PIWOX4c, PmWOX4a, PmWOX4b, PmWUS, PmWOX2, PmWOX3b, $P m W O X 7$ and $P a W U S$ (members of the WUS clade) was elevated in reproductive tissues. The intermediate clade genes, namely PbWOX9, PmWOX9 and PaWOX9, also showed predominant expression in floral buds, except for $P I W O X 9$ which did not show high expression in any of the reproductive tissues. Similarly, the ancient clade genes also showed enhanced expression in the reproductive tissues. $P m W O X 13$ and $P a W O X 13$ showed high expression in floral buds, while $P b W O X 13$ and $P I W O X 13$ showed low expression in the reproductive tissues and high expression in roots. Similarly, PbWOX10 was abundantly expressed in root tissues.

\section{Homology modelling of WOX proteins}

Homology modelling revolutionized the deciphering of protein structure and the mechanism of protein function. Seven WOX proteins (PaWOX9, PaWUS, PbWOX2, PbWOX4, PmWOX13, PmWOX11 and PmWOX10) were selected from $P$. bellina, $P$. modesta and $P$. aphrodite for the secondary and tertiary structure prediction on the basis of their significant expression level in the reproductive tissues and a clade-wise distribution (Table 3, Fig. 6). Alpha-helix and random coils were found to be dominant in the secondary structures (Fig. 6.1A-G). The $3 \mathrm{D}$ structure prediction showed the presence of almost an equal number of alpha-helices in PaWUS, PbWOX2, PbWOX4, PmWOX13, PmWOX11 and PmWOX10 (Fig. 6.2 I-N). The presence of beta-strand was also predicted in a single protein, i.e. PaWOX9 (Fig. 6.2H). The ligand binding analysis showed that PbWOX2, PmWOX10 and PmWOX13 protein sequences have 
A

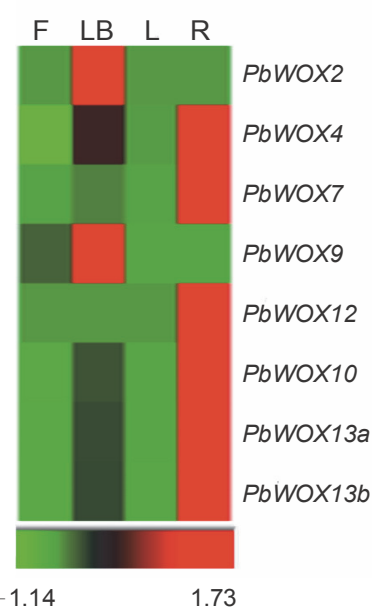

B

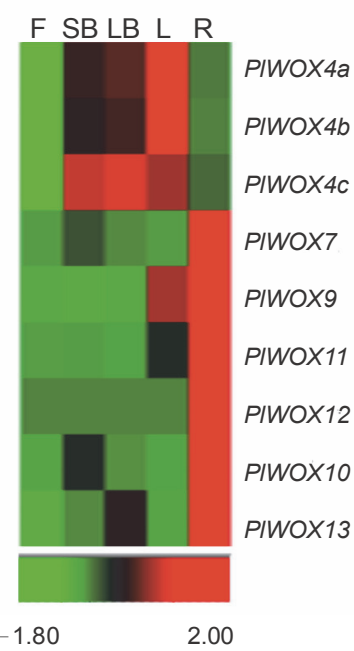

C

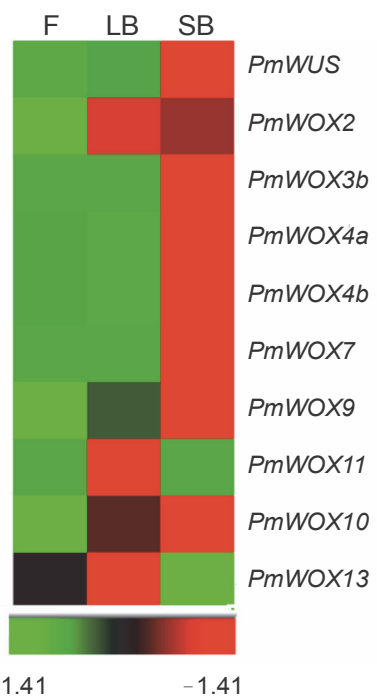

D

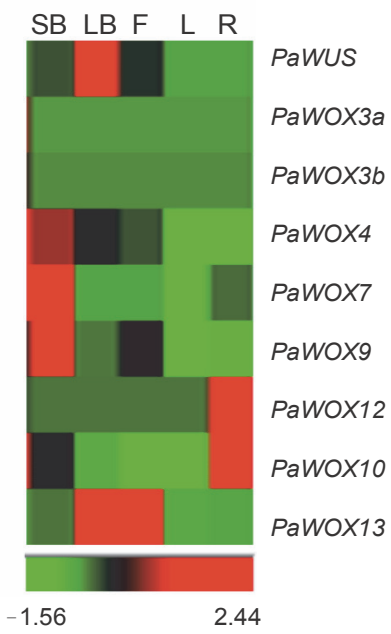

Fig. 5. The expression analysis of the WOX genes in various tissues: A) $P b W O X, \mathrm{~B}) P I W O X, \mathrm{C}) P m W O X$ and D) $P a W O X$ genes (fully opened flower $-\mathrm{F}$, leaf $-\mathrm{L}$, root $-\mathrm{R}$, small bud $-\mathrm{SB}$, large bud $-\mathrm{LB}$, small floral bud $-\mathrm{SB}$, large floral bud $-\mathrm{LB}$ )

Table 3. Prediction of secondary structure and ligand binding sites in selected WOX proteins

\begin{tabular}{l|c|c|c|c|c|l}
\hline \multicolumn{1}{c|}{ Name } & AH & ES & BT & RC & LI & \multicolumn{1}{|c}{ BS } \\
\hline PaWUS & $16.96 \%(39)$ & $6.52 \%(15)$ & $3.48 \%(8)$ & $73.04 \%(168)$ & Zn2+ & 83,87 \\
\hline PaWOX9 & $20.83 \%(65)$ & $13.46 \%(42)$ & $4.81 \%(15)$ & $60.90 \%(190)$ & Mn2+ & $71,97,104,106$ \\
\hline PbWOX2 & $24.67 \%(56)$ & $9.69 \%(22)$ & $5.29 \%(12)$ & $60.35 \%(137)$ & NU & $\begin{array}{l}21,22,24,45,48,70,74,77, \\
81\end{array}$ \\
\hline PbWOX4 & $33.33 \%(60)$ & $14.44 \%(26)$ & $4.44 \%(8)$ & $47.78 \%(86)$ & phosphoric acid & $65,66,70,80,81,82$ \\
\hline PmWOX11 & $28.68 \%(74)$ & $16.28 \%(42)$ & $8.14 \%(21)$ & $46.90 \%(121)$ & Mg2+ & 26,29 \\
\hline PmWOX10 & $52.30 \%(125)$ & $5.44 \%(13)$ & $6.69 \%(16)$ & $35.56 \%(85)$ & NU & $\begin{array}{l}100,101,103,144,144,147, \\
148,150,151,155\end{array}$ \\
\hline PmWOX13 & $45.60 \%(114)$ & $6.80 \%(17)$ & $4.80 \%(12)$ & $42.80 \%(107)$ & NU & $\begin{array}{l}97,98,99,100,101,103,144, \\
147,148,151,155\end{array}$ \\
\hline
\end{tabular}

* AH - alpha helix, RC - random coil, ES - extended strand, BT - beta turn, LI - ligand, BS - binding sites, NU - nucleic acid, $\mathrm{Zn}^{2+}-\mathrm{zinc}$, $\mathrm{Mn}^{2+}-$ manganese ${ }^{2+}, \mathrm{Mg}^{2+}-$ magnesium

specific binding sites for the nucleic acid ligand (Fig. 6.2 J, L, N). PmWOX11, PbWOX4, PaWUS and PaWOX9 contained magnesium $\left(\mathrm{Mg}^{2+}\right)$, phosphoric acid, zinc $\left(\mathrm{Zn}^{2+}\right)$ and manganese $\left(\mathrm{Mn}^{2+}\right)$ binding sites, respectively (Fig. 6.2 M, K, I, H).

\section{Discussion}

The in silico characterization of genes and gene families has become an important research tool in molecular biology to understand various biological pathways involved in growth and development. The WOX gene fa- mily plays a versatile role in embryo pattern formation, organogenesis and florigenesis (van der Graaff et al., 2009; Costanzo et al., 2014). The present study involved protein characterization, topology study and phylogenetic analysis of WOX proteins in four commercially important orchids, namely $P$. aphrodite, $P$. modesta, $P$. bellina and $P$. lueddemanniana. The transcriptomic analysis of $P$. aphrodite, $P$. modesta, $P$. bellina and $P$. lueddemanniana showed that they encode nine, ten, nine and eight full-length WOX proteins, respectively. This finding was comparable to that reported for Phalaenopsis (14), Dendrobium (10) and Apostasia (10) 

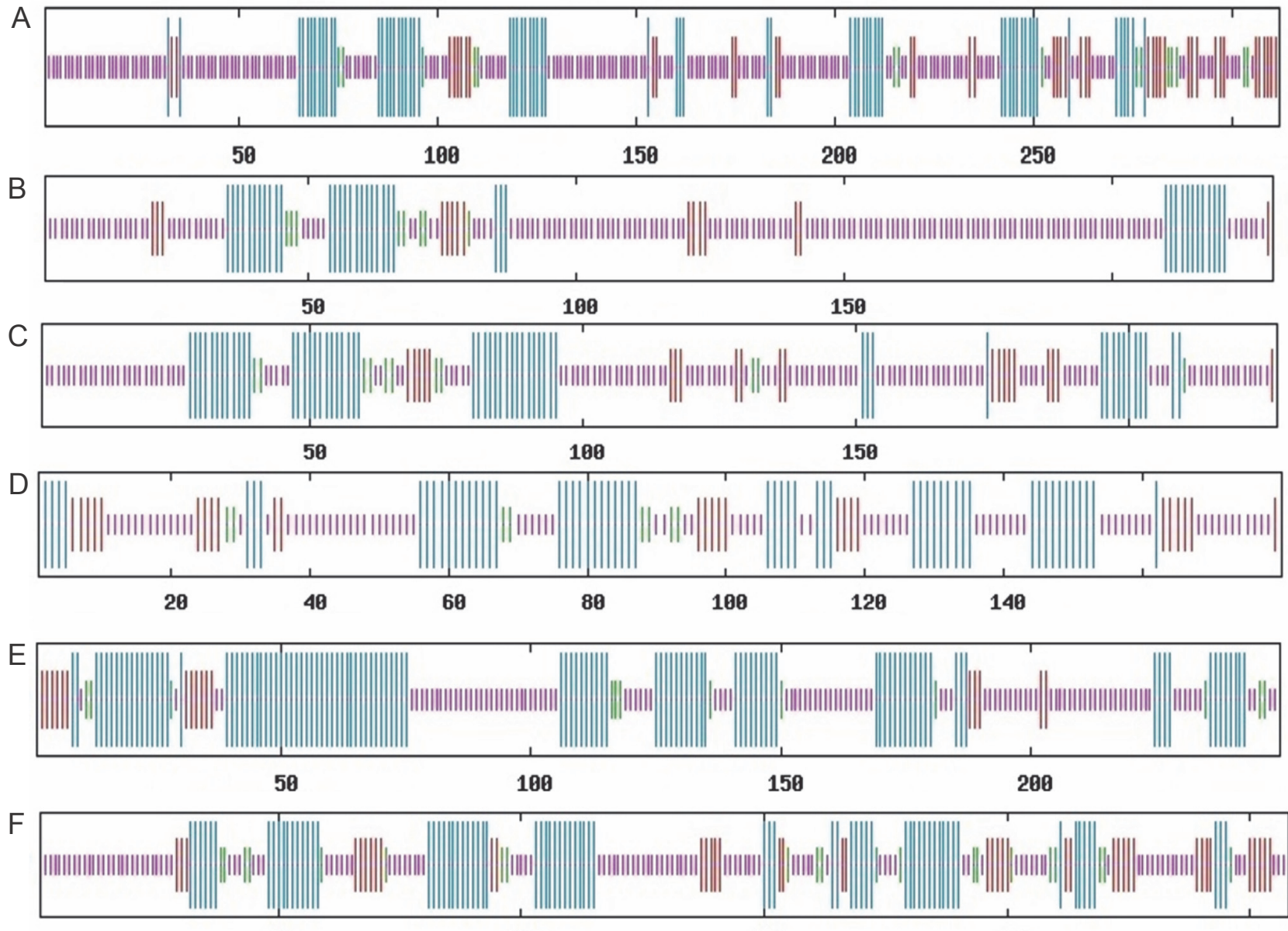

$\begin{array}{llll}50 & 100 & 150 & 200\end{array}$

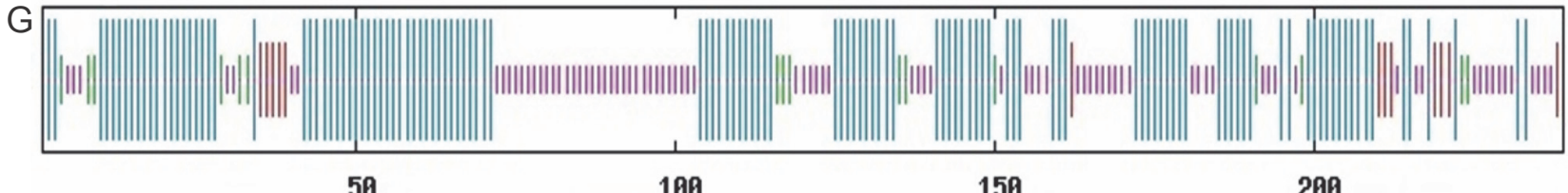

Fig. 6.1. Secondary structure analysis: A) PaWOX9, B) PaWUS, C) PbWOX2, D) PbWOX4, E) PmWOX10, F) PmWOX11 and G) PmWOX13

(Ramkumar et al., 2018, 2020). The multiple sequence alignment showed that all the predicted sequences have a WUSCHEL-related homeobox structure with a helixloop-helix-turn-helix region, which is the major characteristic feature of this gene family. Evolutionary studies showed that the WOX genes of $P$. aphrodite, $P$. modesta, $P$. bellina and $P$. lueddemanniana can be sub-grouped into three clades (Ancient, Intermediate and WUS) in a ratio of $2: 2: 5,2: 2: 6,3: 2: 3$ and $2: 3: 4$, respectively. The tight clustering formed during the construction of the phylogenetic tree along with $P$. equestris and $D$. catenatum showed the common origin of these plants, which is substantiated by their inclusion in the common sub-family Epidendroideae (Freudenstein et al., 2015). The predicted average size and molecular mass of all the PaWOX, PbWOX, PIWOX and PmWOX protein sequences were $250 \mathrm{aa}, 236 \mathrm{aa}, 204$ aa and $232 \mathrm{aa}$ and $27.6 \mathrm{kDa}$, $26.4 \mathrm{kDa}, 23.24 \mathrm{kDa}$ and $20.6 \mathrm{kDa}$, respectively; these values are in sync with those reported for $P$. equestris, $D$. catenatum and $A$. shenzhenica (Ramkumar et al., 2018,2020 ). All the predicted proteins were hydrophilic in nature and located in the nucleus, which is in conformity with previous studies (Sakakibara et al., 2014; Ramkumar et al., 2018, 2020). The duplication event prediction showed the presence of two duplication events in the WUS clade of $P$. lueddemanniana 
$\mathrm{H}$

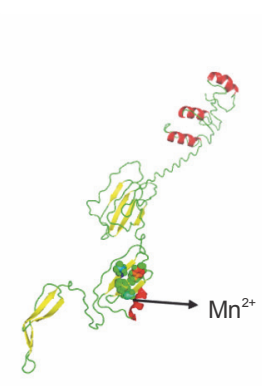

$\mathrm{L}$

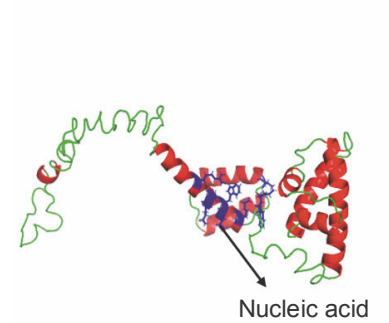

1

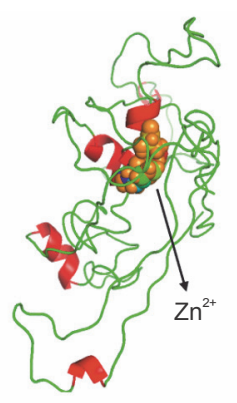

M

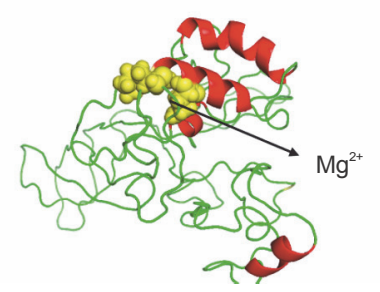

J

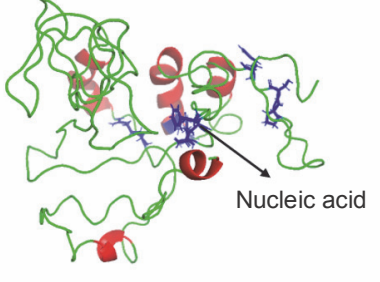

$\mathrm{K}$

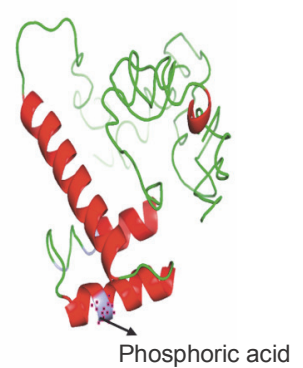

$\mathrm{N}$

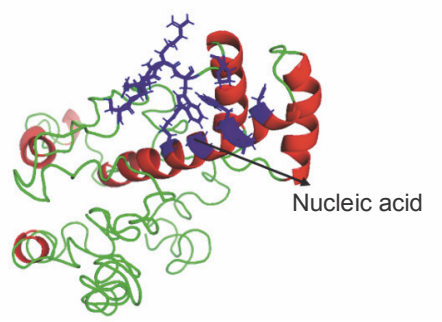

Fig. 6.2. Simulated 3D structures with ligand binding sites: H) PaWOX9, I) PaWUS, J) PbWOX2, K) PbWOX4, L) PmWOX10, M) PmWOX11 and N) PmWOX13

(PIWOX4a, PIWOX4b and PIWOX4c) and one duplication event in the ancient clade of $P$. bellina (PbWOX13a and PbWOX13b). PbWOX13a and PbWOX13b clustered with PeWOX13A/B/C of $P$. equestris, which also has been reported to have two duplication events (PeWOX13A, PeWOX13B and PeWOX13C) (Ramkumar et al., 2018).

The expression pattern analysis of the WOX genes at various developmental stages of plants showed that the WOX gene family has diverse roles in several developmental processes. In our studies, maximum genes were predicted to have good expression in floral organs, which indicates that they might play an active role during floral development as previously reported in many plants (Deyhle et al., 2007; Ikeda et al., 2009; Romera- Branchat et al., 2013; Costanzo et al., 2014). The expression profiling revealed that $\mathrm{PaWUS}$ and PmWUS showed high expression in floral buds, which is in accordance with earlier studies on the WUS gene of $A$. thaliana in which a high expression was related to the regulation of floral patterning by meristem maintenance in floral buds (Ikeda et al., 2009). The predominant expression of the PbWOX13a/b, PIWOX13 and PaWOX13 genes was noted in floral buds and flowers; additionally, abundant expression of $P b W O X 13 a / b$ and $P I W O X 13$ was also noted in roots. Importantly, it was reported that WOX13 plays an important role in the primary and lateral root development, floral transition and replum development in $A$. thaliana (Romera-Branchat et al., 2013); thus, PmWOX2 and PbWOX2 with similar expression levels might be playing similar roles. The PIWOX11 and PIWOX12 genes and their orthologs $P b W O X 12$ and $P a W O X 12$ had preferential expression in all the tested tissues, but abundant expression was noted in roots; this indicates their involvement in root development, as already reported in $A$. thaliana and rice, where these genes function in growth promotion (Hu et al., 2016; Jiang et al., 2017). Protein homology modelling is necessary to understand protein structure, ligand binding and functional mechanisms. In the present work, we selected seven WOX proteins clade wise for the protein homology analysis on the basis of their expression pattern. The predicted 3D structures and ligand binding according to their alignment with top 10 homologous PDB templates of other homeodomain proteins revealed the conserved nature of WOX proteins. The protein-ligand interaction analysis of PaWOX9, PaWUS, PbWOX2, PbWOX4, PmWOX13, PmWOX11 and PmWOX10 showed the presence of five different ligand binding sites, i.e. nucleic acid, $\mathrm{Mg}^{2+}, \mathrm{Mn}^{2+}$, phosphoric acid and zinc. PbWOX2, PmWOX10 and PmWOX13 have nucleic acid ligand binding sites, which supports the finding that these proteins have a DNA binding domain 
(Gehring et al., 1994). However, the binding sites of phosphoric acid in PbWOX4, $\mathrm{Mn}^{2+}$ in PaWOX9, $\mathrm{Zn}^{2+}$ in PaWUS and $\mathrm{Mg}^{2+}$ in PmWOX11 were unexpected (Fig. 6.2H-N). The present study is insufficient to confirm these metal-binding properties of WOX proteins, and an in depth in vitro study is required to provide more supporting evidence.

\section{Conclusions}

As the molecular mechanisms of growth and development are poorly studied in orchids, the present study provides an insight into the WOX gene family in $P$. aphrodite, $P$. modesta, $P$. bellina and $P$. lueddemanniana. This study illustrates that WOX members are conserved in nature at both the sequence and structural levels. They are clustered in their respective clades and show increased expression in floral organs. The present study can help in the functional elucidation of candidate genes for understanding the growth and development in these commercially important orchid species.

\section{Acknowledgements}

MK is thankful to the Department of Science and Technology (DST) for INSPIRE Fellowship for Research Students (File Number: DST/INSPIRE/03/2017/002346). JKS and MK are grateful to the Department of Science and Technology (DST), Government of India for the partial financial support under the Promotion of University Research and Scientific Excellence (PURSE) grant scheme.

\section{Authors' contributions}

JKS designed the work. MK executed the experiments. MK and JKS prepared the manuscript. All authors have read and approved the final manuscript.

\section{Competing interests}

The authors declare that they have no competing interests.

\section{References}

Arroyo-Herrera A., Ku Gonzalez A., Canche Moo R., QuirozFigueroa F.R., Loyola-Vargas V.M., Rodriguez-Zapata L.C., Burgeff D Hondt C., Suárez-Solís V.M., Castaño E. (2008) Expression of WUSCHEL in Coffea canephora causes ectopic morphogenesis and increases somatic embryogenesis. Plant Cell Tissue Organ Cult. 94: 171-180. doi.org/ 10.1007/s11240-008-9401-1

Bailey T.L., Boden M., Buske F.A., Frith M., Grant C.E., Clementi L., Ren J., Li W.W., Noble W.S. (2009) MEME SUITE: tools for motif discovery and searching. Nucl. Acids Res. 37: W202-W208. doi.org/10.1093/ nar/gkp335
Bouchabké-Coussa O., Obellianne M., Linderme D., Montes E., Maia-Grondard A., Vilaine F., Pannetier C. (2013) WUSCHEL overexpression promotes somatic embryogenesis and induces organogenesis in cotton (Gossypium hirsutum L.) tissues cultured in vitro. Plant Cell Rep. 32: 675-686. doi.org/10.1007/s00299-013-1402-9

Breuninger H., Rikirsch E., Hermann M., Ueda M., Laux T. (2008) Differential expression of WOX genes mediates apical-basal axis formation in the Arabidopsis embryo. Develop. Cell 14: 867-876. doi.org/10.1016/j.devcel. 2008.03.008

Chao Y.T., Yen S.H., Yeh J.H., Chen W.C., Shih M.C. (2017) Orchidstra 2.0-a transcriptomics resource for the orchid family. Plant Cell Physiol. 58: e9. doi.org/10.1093/pcp/ pcw220

Corpet F. (1988) Multiple sequence alignment with hierarchical clustering. Nucl. Acids Res. 16: 10881-10890. doi.org/10.1093/nar/16.22.10881

Costanzo E., Trehin C., Vandenbussche M. (2014) The role of WOX genes in flower development. Ann. Bot. 114: 1545-1553. doi.org/10.1093/aob/mcu123

DeLano W.L. (2002) Pymol: an open-source molecular graphics tool. CCP4 Newsletter Protein Crystallogr. 40: 82-92.

Deveaux Y., Toffano-Nioche C., Claisse G., Thareau V., Morin H., Laufs P., Moreau H., Kreis M., Lecharny A. (2008) Genes of the most conserved WOX clade in plants affect root and flower development in Arabidopsis. BMC Evolut. Biol. 8: 291. doi.org/10.1186/1471-2148-8-291

Deyhle F., Sarkar A.K., Tucker E.J., Laux T. (2007) WUSCHEL regulates cell differentiation during anther development. Develop. Biol. 302: 154-159. doi.org/ 10.1016/j.ydbio.2006.09.013

Edgar R.C. (2004) MUSCLE: multiple sequence alignment with high accuracy and high throughput. Nucl. Acids Res. 32: 1792-1797. doi.org/10.1093/nar/gkh340

Freudenstein J.V., Chase M.W. (2015) Phylogenetic relationships in Epidendroideae (Orchidaceae), one of the great flowering plant radiations: progressive specialization and diversification. Ann. Bot. 115: 665-681. doi.org/ 10.1093/ aob/mcu253

Gasteiger E., Hoogland C., Gattiker A., Duvaud S., Wilkins M.R., Appel R.D., Bairoch A. (2005) Protein identification and analysis tools on the ExPASy server. Springer.

Gehring W.J., Affolter M., Burglin T. (1994) Homeodomain proteins. Ann. Rev. Biochem. 63: 487-526. doi.org/ 10.1146/annurev.bi.63.070194.002415

Haecker A., Groß-Hardt R., Geiges B., Sarkar A., Breuninger H., Herrmann M., Laux T. (2004) Expression dynamics of WOX genes mark cell fate decisions during early embryonic patterning in Arabidopsis thaliana. Development 131: 657-668. doi:10.1242/dev.00963

Horton P., Park K.J., Obayashi T., Fujita N., Harada H., Adams-Collier C.J., Nakai K. (2007) WoLF PSORT:protein localization predictor. Nucl. Acids Res. 35: 585-587. doi.org/10.1093/nar/gkm259 
Hu X., Xu L. (2016) Transcription factors WOX11/12 directly activate WOX5/7 to promote root primordia initiation and organogenesis. Plant Physiol. 172: 2363-2373. doi.org/ 10.1104/pp.16.01067

Ikeda M., Mitsuda N., Ohme-Takagi M. (2009) Arabidopsis WUSCHEL is a bifunctional transcription factor that acts as a repressor in stem cell regulation and as an activator in floral patterning. Plant Cell 21: 3493-3505. doi.org/ 10.1105/tpc.109.069997

Jha P., Ochatt S.J., Kumar V. (2020) WUSCHEL: a master regulator in plant growth signaling. Plant Cell Rep. 1: 1-4. doi.org/10.1007/s00299-020-02511-5

Jiang W., Zhou S., Zhang Q., Song H., Zhou D.X., Zhao Y. (2017) Transcriptional regulatory network of WOX11 is in volved in the control of crown root development, cytokinin signals, and redox in rice. J. Exp. Bot. 68: 2787-2798. doi.org/10.1093/jxb/erx153

Kong D., Hao Y., Cui H. (2016) The WUSCHEL related homeobox protein WOX7 regulates the sugar response of lateral root development in Arabidopsis thaliana. Mol. Plant 9: 261-270. doi.org/10.1016/j.molp.2015. 11.006

Krogh A., Larsson B., von Heijne G., Sonnhammer E.L. (2001) Predicting transmembrane protein topology with a hidden Markov model: application to complete genomes. J. Mol. Biol. 305: 567-580. doi.org/10.1006/jmbi.2000. 4315

Kumar S., Stecher G., Tamura K. (2016) MEGA7: Molecular Evolutionary Genetics Analysis Version 7.0 for Bigger Datasets. Mol.Biol.Evol.33: 1870-1874. doi.org/10.1093/ molbev/msw054

Laux T., Mayer K.F., Berger J., Jurgens G. (1996) The WUSCHEL gene is required for shoot and floral meristem integrity in Arabidopsis. Development 122: 87-96. doi.org/ 10.1371/journal.pone.0038161

Lian G., Ding Z., Wang Q., Zhang D., Xu J. (2014) Origins and evolution of WUSCHEL-related homeobox protein family in plant Kingdom. Sci. World J. 2014: 1-12. doi.org/ $10.1155 / 2014 / 534140$

Liu J., Sheng L., Xu Y., Li J., Yang Z., Huang H., Xu L. (2014) WOX11 and 12 are involved in the first-step cell fate transition during de novo root organogenesis in Arabidopsis. Plant Cell 26: 1081-1093. doi.org/10.1105/tpc. 114.122887

Lohmann J.U., Hong R.L., Hobe M., Busch M. A., Parcy F., Simon R., Weigel D. (2001) A molecular link between stem cell regulation and floral patterning in Arabidopsis. Cell 105: 793-803. doi.org/10.1016/S0092-8674(01) 00384-1

Matsumoto N., Okada K. (2001) A homeobox gene, PRESSED FLOWER, regulates lateral axis-dependent development of Arabidopsis flowers. Genes Develop. 15: 3355-3364. www.genesdev.org/cgi/doi/10.1101/gad.931001.

Mayer K.F., Schoof H., Haecker A., Lenhard M., Jurgens G., Laux T. (1998) Role of WUSCHEL in regulating stem cell fate in the Arabidopsis shoot meristem. Cell 95: 805-815. doi.org/10.1016/S0092-8674(00)81703-1

Mortazavi A., Williams B.A., McCue K., Schaeffer L., Wold B. (2008) Mapping and quantifying mammalian transcripto- mes by RNA-Seq. Nature Meth. 5: 621-628. doi.org/ 10.1038/nmeth. 1226

Nardmann J., Ji J., Werr W., Scanlon M.J. (2004) The maize duplicate genes narrow sheath1 and narrow sheath2 encode a conserved homeobox gene function in a lateral domain of shoot apical meristems. Development 131: 2827-2839. doi: 10.1242/dev.01164

Oshchepkova E.A., Omelyanchuk N.A., Savina M.S., Pasternak T., Kolchanov N.A., Zemlyanskaya E.V. (2017) Systems biology analysis of the WOX5 gene and its functions in the root stem cell niche. Russ. J. Gen. Appl. Res. 7: 404-420. doi.org/10.1134/S2079059717040086

Park S.O., Zheng Z., Oppenheimer D.G., Hauser B.A. (2005) The PRETTY FEW SEEDS2 gene encodes an Arabidopsis homeodomain protein that regulates ovule development. Development 132: 841-849. doi: 10.1242/dev. 01654

Petersen T.N., Brunak S., von Heijne G., Nielsen H. (2011) SignalP 4.0: discriminating signal peptides from transmembrane regions. Nature Meth. 8: 785-786. doi.org/ 10.1038/nmeth.1701

Ramkumar T.R., Kanchan M., Upadhyay S.K., Sembi J.K. (2018) Identification and characterization of WUSCHELrelated homeobox (WOX) gene family in economically important orchid species Phalaenopsis equestris and Dendrobium catenatum. Plant Gene 14: 37-45. doi.org/ 10.1016/ j.plgene.2018.04.004

Ramkumar T.R., Kanchan M., Sembi J.K. (2020) Genome wide characterization of WUSCHEL-related homeobox (WOX) gene family in Apostasia shenzhenica, a primeval orchid. Plant Sci. Today 7: 164-171. doi.org/10.14719/pst.2020. 7.2.703

Rebocho B.A., Bliek M., Kusters E., Castel R., Procissi A., Roobeek I., Souer E., Koes R. (2008) Role of EVERGREEN in the development of the Cymose petunia inflorescence. Develop. Cell 15: 437-447. doi.org/10.1016/j.devcel.2008. 08.007

Romera-Branchat M., Ripoll J.J., Yanofsky M.F., Pelaz S. (2013) The WOX13 homeobox gene promotes replum formation in the Arabidopsis thaliana fruit. Plant J. 73: 37-49. doi.org/10.1111/tpj.12010

Rupps A., Raschke J., Rummler M., Linke B., Zoglauer K. (2016) Identi cation of putative homologs of Larix decidua to babyboom (BBM), leafy cotyledon1 (LEC1), WUSCHELrelated homeobox2 (WOX2) and somatic embryogenesis receptor-like kinase (SERK) during somatic embryogenesis. Planta 243: 473-488. doi.org/10.1016/j.gene.2018.12.049

Sakakibara K., Reisewitz P., Aoyama T., Friedrich T., Ando S., Sato Y., Tamada Y., Nishiyama T., Hiwatashi Y., Kurata T., Ishikawa M. (2014) WOX13-like genes are required for reprogramming of leaf and protoplast cells into stem cells in the moss Physcomitrella patens. Development 141: 1660-1670. https://doi.org/10.1242/dev.097444

Sapay N., Guermeur Y., Deléage G. (2006) Prediction of amphipathic in-plane membrane anchors in monotopic proteins using a SVM classifier. BMC Bioinformatics 7: 255. doi.org/10.1093/bioinformatics/btk052 
Scanlon M.J., Schneeberger R.G., Freeling M. (1996) The maize mutant narrow sheath fails to establish leaf margin identity in a meristematic domain. Development 122: 1683-1691.

Schultz J., Copley R.R., Doerks T., Ponting C.P., Bork P. (2000) SMART: a web-based tool for the study of genetically mobile domains. Nucl. Acids Res. 28: 231-234. doi.org/10.1093/nar/28.1.231

Seo J., Gordish-Dressman H., Hoffman E.P. (2006) An interactive power analysis tool for microarray hypothesis testing and generation. Bioinformatics 22: 808-814. doi.org/ 10.1093/bioinformatics/btk052

Sigrist C.J.A., de Castro E., Cerutti L., Cuche B.A., Hulo N., Bridge A., Bougueleret L., Xenarios I. (2012) New and continuing developments at PROSITE. Nucl. Acids Res. 41: 344-347. doi.org/10.1093/nar/gks1067

Su C.L., Chao Y.T., Alex Chang Y.C., Chen W.C., Chen C.Y., Lee A.Y., Shih M.C. (2011) De novo assembly of expressed transcripts and global analysis of the Phalaenopsis aphrodite transcriptome. Plant Cell Physiol. 52: 1501-1514. doi.org/10.1093/pcp/pcr097

Suer S., Agusti J., Sanchez P., Schwarz M., Greb T. (2011) WOX4 imparts auxin responsiveness to cambium cells in Arabidopsis. Plant Cell 23: 3247-3259. doi.org/10.1105/ tpc.111.087874
Ueda M., Zhang Z., Laux T. (2011) Transcriptional activation of Arabidopsis axis patterning genes WOX8/9 links yygote polarity to embryo development. Develop. Cell 20: 264-270. doi.org/10.1016/j.devcel.2011.01.009

van der Graaff E., Laux T., Rensing S.A. (2009) The WUS homeobox-containing (WOX) protein family. Genome Biol. 10: 248. doi.org/10.1186/gb-2009-10-12-248

Wu P.H., Chang C.N.D. (2012) Cytokinin treatment and flower quality in Phalaenopsis orchids: Comparing N-6-benzyladenine, kinetin and 2-isopentenyl adenine. Afr. J. Biotech. 11: 1592-1596. doi.org/10.5897/AJB11.2472

Yang J., Yan R., Roy A., Xu D., Poisson J., Zhang Y. (2015) The I-TASSER Suite: protein structure and function prediction. Nature Meth. 12: 7-8. doi.org/10.1038/nmeth. 3213

Yu C.S., Chen Y.C., Lu C.H., Hwang J.K. (2006) Prediction of protein subcellular localization. Prot. Struct. Func. Bioinform. 64: 643-651. doi.org/10.1002/prot.21018

Zhang X., Zong J., Liu J., Yin J., Zhang D. (2010) Genome-wide analysis of WOX gene family in rice, sorghum, maize, Arabidopsis and poplar. J. Integr. Plant Biol. 52: 1016-1026. dx.doi.org/10.1111/j.1744-7909.2010.00982.x

Zuo J., Niu Q.W., Frugis G., Chua N.H. (2002) The WUSCHEL gene promotes vegetative-to-embryonic transition in Arabidopsis. Plant J. 30: 349-359. doi.org/10.1046/ j.1365313X.2002.01289.x 